\title{
Research on the Construction of Public Service System of Community
}

\section{Sports in China}

\author{
Hao Cui ${ }^{1, a}$ \\ ${ }^{1}$ Dalian Maritime University, Dalian, Liaoning, China, 116026 \\ ${ }^{a}$ email
}

Keywords: Community Sports, Public Service System, Sports Power

\begin{abstract}
Community sports have developed very rapid in recent years. The public service system of community sports plays an important role in the process of the construction of a sports power. However, due to the constraints of time and experience, the construction of public service system of community sports has some problems, which are the insufficient construction funds, low participation level, deficient professional talents and imperfect management system. This paper analyzes the above problems, and puts forward the corresponding countermeasures in order to provide some references for the relevant researchers.
\end{abstract}

\section{Introduction}

Sports public service is a type of sports service with the nature of public goods. In the key period comprehensively building a well-off society, sports as a satisfy the people's physical health, mental and physical pleasure, improve quality of life and cultural phenomenon, community sports by unprecedented attention, has become a hot issue in the sports life and socialization. With the development of China's economy, people's material living standards improve, more and more people to a variety of service requirements are higher and higher, community sports public service system is no exception. Community public service system is refers to the governments at all levels in order to protect the urban community residents basic sports rights and interests, to meet the growing demand for sports service and invest in community public sports facilities, public sports organization network, the system of mass sports activities, social welfare body education instructors, fitness guidance and information service system for support and operation platform for the basic framework of coverage throughout the city community sports fitness service system.

The construction of community sports public service system is an important part of the construction of sports public service system in our country. With the comprehensive construction well-off society in the critical period and deepening reform and opening up and accelerate the transformation of the mode of economic development in the crucial period, on the one hand, leisure time of urban residents generally increased, development is also generally increasing the consciousness, residents of leisure and entertainment, social, fashion sports, self-realization demand and rising. On the other hand, the development of urbanization is faster and faster, and many rural population changes like the city population, thus making the city scale unceasingly expands. At the same time, due to the pressure of modern life, lifestyle and environmental pollution factors, so that a variety of cardiovascular and cerebrovascular diseases and a variety of mental illness has become a major threat to the health of the residents. These new changes create the conditions of some community sports public service system. The model to meet the needs of the residents of the 
community sports become an important part of the community sports public service system construction.

\section{Problems of Public Service System of Community Sports}

At present, the construction of community sports public service system in our country is still in the initial stage because of short time and experience. The public service system of community sports has many problems.

Insufficient Construction Funds. Sufficient funds for sports activities are the guarantee for the comprehensive development of community sports public service. Government expenditure is the main source of funds for community sports activities. The single source of community sports funding has seriously restricted the establishment of community sports public service system. Community per capita share of sports venues is low and uneven distribution. Community sports public facilities with expensive is not perfect, the venues and facilities management poor, aging facilities, stolen, destroyed, causing serious, if not promptly update maintenance, easy in the use of unnecessary sports injury accident occurred in the process. In addition to the country's public financial investment in this area, on the one hand, community managers cannot give full play to the initiative, the initiative to seek funding sources. On the other hand, enterprises and institutions are driven by the interests of sports activities into the selective, small scale community sports activities to support the possibility of small and unstable. Community sports club model has failed to make full use of sports clubs annual expenditure will be deficit, failed to form a self-profitable results.

Low Participation Level. The common sports facilities of community sports are fitness path, basketball courts and table tennis tables, etc. There is a small part of the conditions of the community also has paid services to the gym or health club, the public sports facilities for community residents to participate in physical exercise, provide all kinds of material carrier. There is no formation of community sports aggregation force, in the enthusiasm of any need to be improved. Community residents to professional sports, mass sports competition performance and other activities interest is not high, and more concerned about is and their related most directly, the most realistic and the most basic sports events, of high level, high strength and high consumption of large or professional sports activities need not. Overall community sports events are not rich enough, the scale is small, residents participate in the enthusiasm is not high. Many activities are difficult to carry out a good. Community sports managers need to pay more attention to the work, starting from the specific needs of the community residents, the organization diversified, people first, the convenience of sports and fitness activities. Community residents seldom participate in daily physical activity, and the daily sports activities in order to enable residents real exercise his body, his cheerful mood, and festival sports activities is mainly community managers is held to celebrate the festival activities. The festival is over, and it's hard to keep it down. The phenomenon of the community participation is not high.

Deficient Professional Talents. Communities are equipped with corresponding sports management personnel and social sports instructors. These talents are seldom the graduates of physical education of normal colleges and universities mainly because of the poor conditions and low pay of community. Overall, whether in quantity or in quality, there is a significant gap between the actual demand, there is a lack of professional knowledge, unreasonable structure, part-time staff, poor sense of responsibility and so on. In these social sports instructors, no one has systematically studied the professional knowledge of physical education, only a few are due to work needs to learn some of the single discipline. Although most of them have some work experience, there is no lack of enthusiasm for community sports, but to rely on them to carry out the true sense of scientific 
fitness guidance. At the same time, due to the lack of appropriate incentive system of community sports, in practical work, social sports instructors to plenty of time and enthusiasm of community residents' sports activities of effective guidance, often in a state not as, sports and fitness guide the work a name without a solid. The lack of community sports instructors also hinders the construction of community sports public service system.

Imperfect Management System. The related management department to carry out the work of community sports are not their own things, mutual shirk responsibility. Thus resulting in the community sports services to carry out the difficulties, it is difficult to form a sound management system. Community sports management responsibilities are still not clear. Community Sports Bureau cadres believe that the community sports public service system construction is the thing of the neighborhood, and community managers believe that the community sports public service system construction is the main sports bureau. Thus the community sports service in manpower, material and financial resources will be very difficult to solve; also relatively simple community sports service organization form, the organization and management of each project is not perfect, community sports service organization and management system has not formed the pattern of network. However, the city sports facilities are provided by the government, the activities of the organization mostly by the government tube office. The government should not have a handle, thus forming a single main supply. And today is a market driven society, government monopoly has not adapted to the needs of the development of the times, the government should not products directly to the producer but product providers, managers and transforming government functions and accurate positioning.

\section{Countermeasures of Construction of Public Service System of Community Sports}

Broaden Fund Channels. With the development of regional economic integration and social progress, the construction and improvement of community sports public service system put forward new challenges and requirements. Under the condition of market economy, the community sports public service should be put to the market competition, but this kind of investment and competition is not equal in the culture market specializing in profit of the entertainment business units. To carry out community sports public service activities must have a stable material resources, financial resources and human resources to ensure. In addition to actively raise funds for external support, but also for all walks of life in the community support, fully explore the internal potential. And the development of resources must be reliable, stable, and many aspects of. How to raise and utilize the resources is the skill that the community sports workers must master. Governments at all levels should formulate and implement community sports public service economy policy, to government led, multi input as the motive force of the development, to the public cultural undertakings for the direction of development, encourage and promote people, especially the non-state economic investment and community cultural activities, fully develop and use around the deep historical and cultural heritage and sports resources, encourage private capital in the construction of community sports public service system and perfect to promote community sports public service industry optimization and upgrading.

Raise Participation Level. Carrying out diversified community sports service is an important way to promote public participation in community sports. The diversification of urban and rural community sports public service content and form, as far as possible to take all factors into consideration, to meet the residents with different levels and different occupations of sports public service needs. Along with the deepening of the reform of the public service type government, the supply of urban and rural community sports public service should change the situation of single 
subject, government monopoly, through policy guidance, to attract social and folk sports resources to participate in the supply of urban and rural community sports public service, the implementation of urban and rural community sports public service by the government leading to society and government, nonprofit organizations and commercial organizations work together to provide change, to increase the total supply of, and enhance the supply efficiency, in order to achieve the diversification of the urban and rural community sports public service supply of the main. The diversification of public service demand of urban and rural communities will require the diversification of content and form. With the continuous deepening of the economic level of rapid development and the national fitness campaign, the majority of community residents are increasingly aware of the importance of physical exercise. We should carry out the diversification of community sports public service, according to the different characteristics of different regions and different groups to meet the residents in all aspects of the physical fitness needs.

Strengthen Talents Construction. Social sports instructors have important guiding role in the construction of sports public service system. It is an important support and guarantee for the implementation of sports public service, and the quality of professional quality level determines the quality of service. In view of the currently community population cardinal number is big, the lack of venues and sports facilities, sports funding and salary is limited. It is difficult to attract full-time service personnel to participate in the work of sports public service. Therefore, to strengthen community social sports instructors are considered from the selection and training of community residents. Personnel selection are: school physical education teachers, willing to engaged in the work of the social sports instructors of university graduates, physical quality good community sports volunteers. The unified training of social sports instructors for the selection of personnel, so that it has a social sports instructor qualification certificate. Closer integration resources to carry out regional activities of mass sports culture, to all levels and in all sectors of the call people gather together, with a healthy and positive with the effectiveness of guiding ideology influence people's mind and soul. We should advocate a healthy and civilized way of life to improve and enhance people's quality of life.

Improve Management System. Community sports public service system to establish the process of government responsibility positioning is not accurate, resulting in the community sports public service system is difficult to carry out. The government plays a leading role in the system construction process. Rationally define the function and orientation of the government in the supply of sports public service, and give full play to the role of government guidance and service. As the market main body of officials to fair and reasonable to deal with some of the disputes of interests, only through the change of government functions can realize constructing China street community sports public service system. The government should strengthen the construction of the city community sports public service system of the importance of understanding, the construction of urban community sports public service system into the urban planning. Relevant departments should develop the community sports work of policies, laws and regulations to regulate the road of public service sports in communities in China.

\section{Conclusion}

As an important part of the national sports service system, public service system of community sports plays an important role in the process of China's sports power construction. In the course of the construction of the community sports public service system in our country, we should make every effort to broaden the financing channels, enhance the residents participation, strengthen the 
construction of sports talents and optimize the management mechanism to meet the people's growing demand for sports.

\section{References}

[1] Zhong Jun, Shandong Sports Science \& Technology, Vol. 36 (2014) No 1, p.114-118

[2] Liu Jianxue, Journal of Chongqing Technology and Business University (Natural Science Edition), Vol. 33 (2016) No 1, p.116-120

[3] Hu Yin, Journal of Beijing Sport University, Vol. 32 (2009) No 5, p.12-15

[4] Liu Zhicheng, Journal of Sports and Science, Vol. 33 (2012) No 4, p.75-80 